\title{
The effect of using different EOS in modelling the $\alpha$ Centauri binary system.
}

\author{
A. Miglio \\ Institut d'Astrophysique et Géophysique de l'Université de Liège, Belgium
}

\begin{abstract}
In this preliminary study we investigate the effects of using different equations of state (CEFF and OPAL) in the calibration of the binary system $\alpha$ Centauri. Constraints coming from the detection of acoustic oscillations in $\alpha$ Centauri A and B are included in the modelling.
\end{abstract}

\section{INTRODUCTION}

The visual binary system $\alpha$ Centauri represents a promising target to test our understanding of stellar structure and evolution due to its numerous and stringent observational constraints, including the recent detection of acoustic oscillations frequencies in both components of the system [4], [6].

\section{MODELLING $\alpha$ CENTAURI}

The calibration of the system consists in defining a goodness-of-fit measurement $\left(\chi^{2}\right)$, that includes both seismic and non-seismic constraints weighted with their observational uncertainties, and then minimizing the $\chi^{2}$ using a Levenberg-Marquardt optimization procedure. Models for components A and B, computed with the same initial chemical composition, are fitted to their observational constraints at the same age. We take as observational constraints the small $(\delta v)$ and large $(\Delta v)$ frequency separations (see e.g. [8]) derived from the observed oscillation frequencies by [4] and [6] as well as the effective temperature, luminosity and metallicity (Table 1). A more detailed description of the calibration procedure and of the choice of observational constraints will be reported in [16].

We take as free parameters the age, the initial chemical composition (X,Z) and the mixing length parameter for each component: $\alpha_{A}$ and $\alpha_{B}$. The masses are fixed and taken equal to the values given by [18] $\left(\mathrm{M}_{\mathrm{A}}=1.105 \mathrm{M}_{\odot}\right.$ and $\left.\mathrm{M}_{\mathrm{B}}=0.934 \mathrm{M}_{\odot}\right)$. We have also allowed a variation of the masses within their error bars [16], but its effect is not relevant while studying the effect of different EOS on the calibration of the system.

The stellar models are computed using CLES (Code Liégeois d'Evolution Stellaire). The opacity tables are those of OPAL96 [13] complemented at $T<6000$ with Alexander 

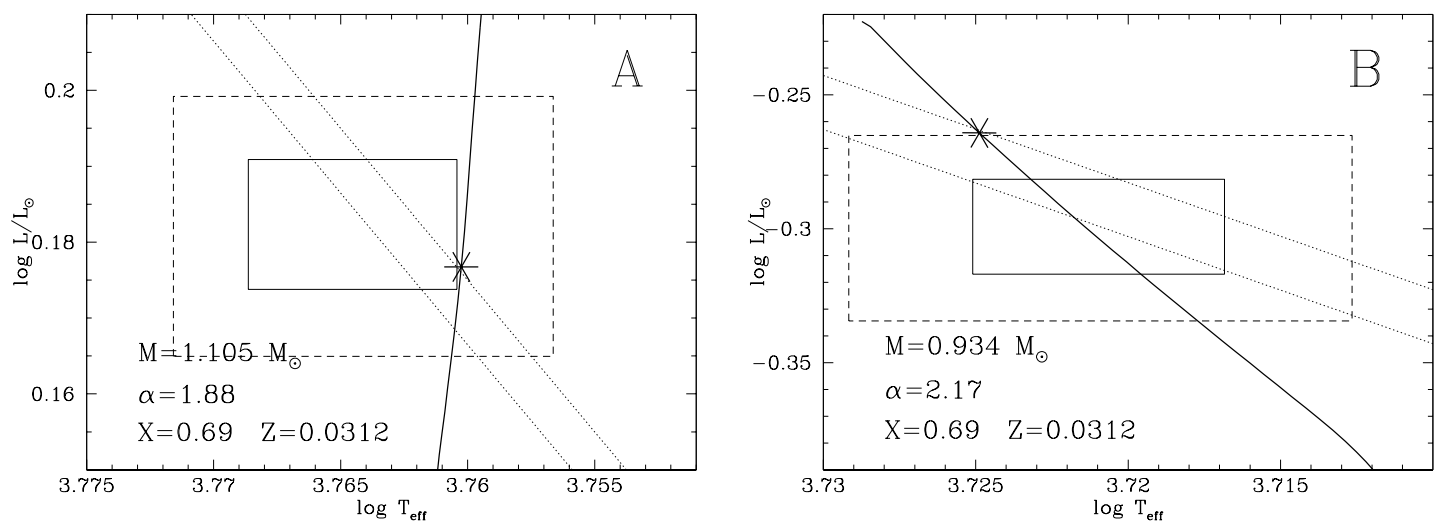

FIGURE 1. Position of models $\mathrm{A} 0$ and $\mathrm{B} 0$ in the HR diagram with $1 \sigma$ and $2 \sigma$ error boxes in luminosity, effective temperature and radius ( $2 \sigma$, assuming the values determined by [14]).
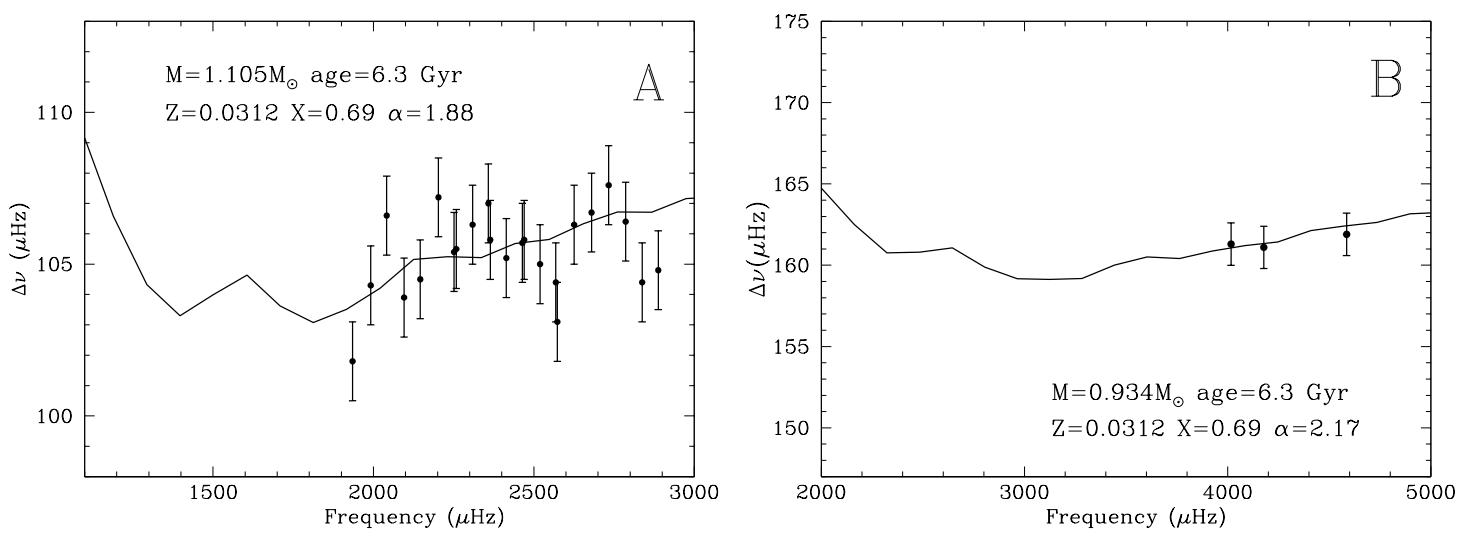

FIGURE 2. Comparison between observed and predicted (model A0 and B0) large frequency separation $\Delta v$ for $\alpha$ Cen A and B.

and Ferguson opacities [1]. The solar Z-distribution from [12] is adopted in the opacities and in the equation of state. Nuclear reaction rates are taken from [7] and the screening factor from [20]. Convective zones are treated with the classical mixing length theory [3] with the formulation by [10]; atmospheric boundary conditions [15] are applied at $T=T_{\text {eff. }}$ The code includes microscopic diffusion of $\mathrm{H}, \mathrm{He}$ and $\mathrm{Z}$ using the subroutine by [21]. The equation of state can be chosen among CEFF [9] and OPAL [19].

Fig. 11 shows the HR diagram location of the $\alpha$ Cen A and B models that best fit the classical $\left(T_{\text {eff }}, \mathrm{L}\right)$ and seismic observables (hereafter A0 and B0, see Table 2); in Figure 2 and 3 we plot the corresponding large and small separations. 

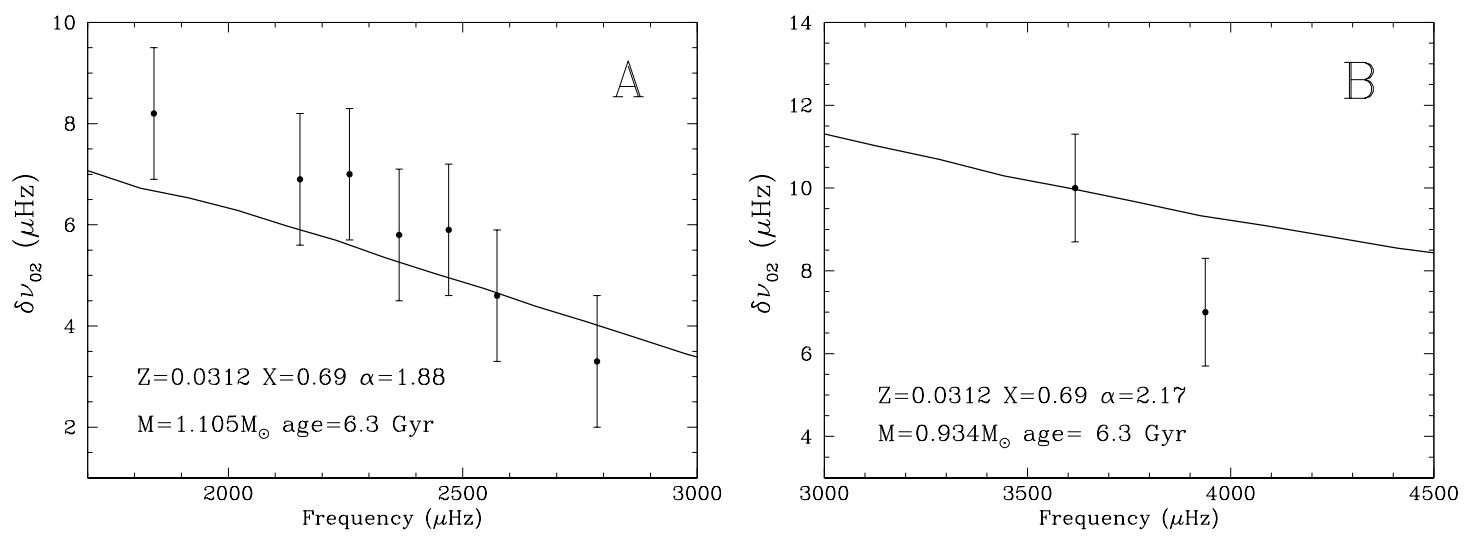

FIGURE 3. Observed versus predicted small frequency separation $(\delta v)$.

TABLE 1. Non-asteroseismic constraints assumed in the calibration

\begin{tabular}{cccc}
\hline & $\mathrm{A}$ & $\mathrm{B}$ & Ref \\
\hline$T_{\text {eff }}(\mathrm{K})$ & $5810 \pm 50$ & $5260 \pm 50$ & {$[17]$} \\
$\mathrm{L} / \mathrm{L} \odot$ & $1.522 \pm 0.030$ & $0.503 \pm 0.020$ & {$[11]$} \\
$(Z / X)_{\mathrm{S}}$ & $0.039 \pm 0.006$ & $0.039 \pm 0.006$ & {$[22]$} \\
\hline
\end{tabular}

INTRINSIC DIFFERENCE

In order to analyze the differences between CEFF and OPAL equations of state, we take the internal structure $(T-\rho)$ of the best models (A0 and B0) calibrated using OPAL and we estimate $c^{2}$ and $\Gamma_{1}$ using CEFF. In Fig. 4 we show the internal structure of the models A0 and B0 as well as the structure of a solar model for comparison.

The differences between sound speed and first adiabatic exponent, due solely to the use of a different EOS, are shown in Fig. 5]and 6. These differences are of the same order of those predicted in solar models [2], they appear to be larger in the lower-mass model B0 than in model A0 and are mainly located in the hydrogen and helium ionization regions. Such a small difference in the internal structure of a model propagates in a variation of the observables of each model (e.g. effective temperature, luminosity) that could be easily compensated by a re-adjustment of the free parameters in our modelling. Slightly changing the initial chemical composition, the mixing length parameter or the age, would easily let the models computed with different equations of state satisfy the observational constraints.

TABLE 2. Model parameters for reference models $\mathrm{A} 0$ and $\mathrm{B} 0$.

\begin{tabular}{cccccc}
\hline Model & M/M $\odot$ & $\alpha$ & Age $(\mathrm{Gyr})$ & $X$ & $Z$ \\
\hline A0 & 1.105 & 1.88 & 6.3 & 0.690 & 0.0312 \\
B0 & 0.934 & 2.17 & 6.3 & 0.690 & 0.0312 \\
\hline
\end{tabular}




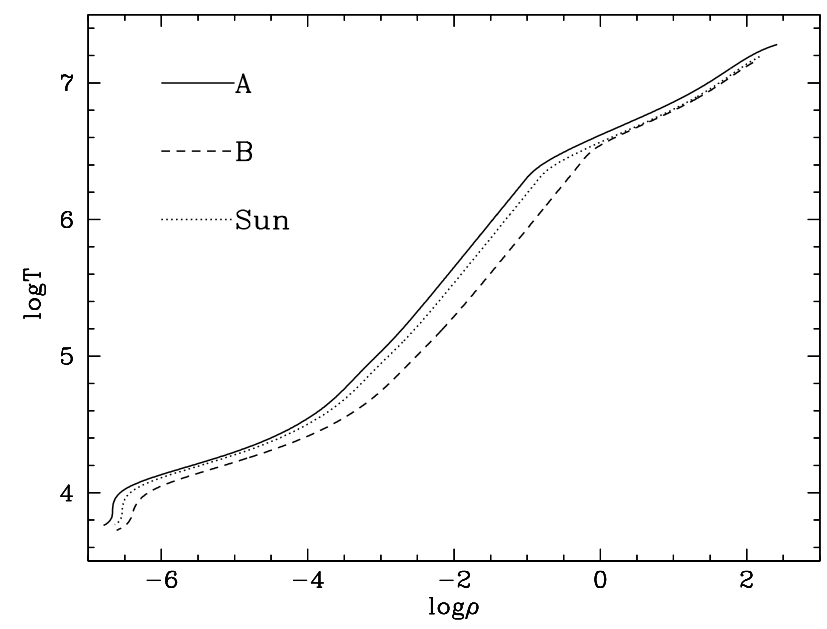

FIGURE 4. Internal temperature-density profile of reference models $\mathrm{A} 0$ and $\mathrm{B} 0$ compared to the profile of a solar model (dotted line).
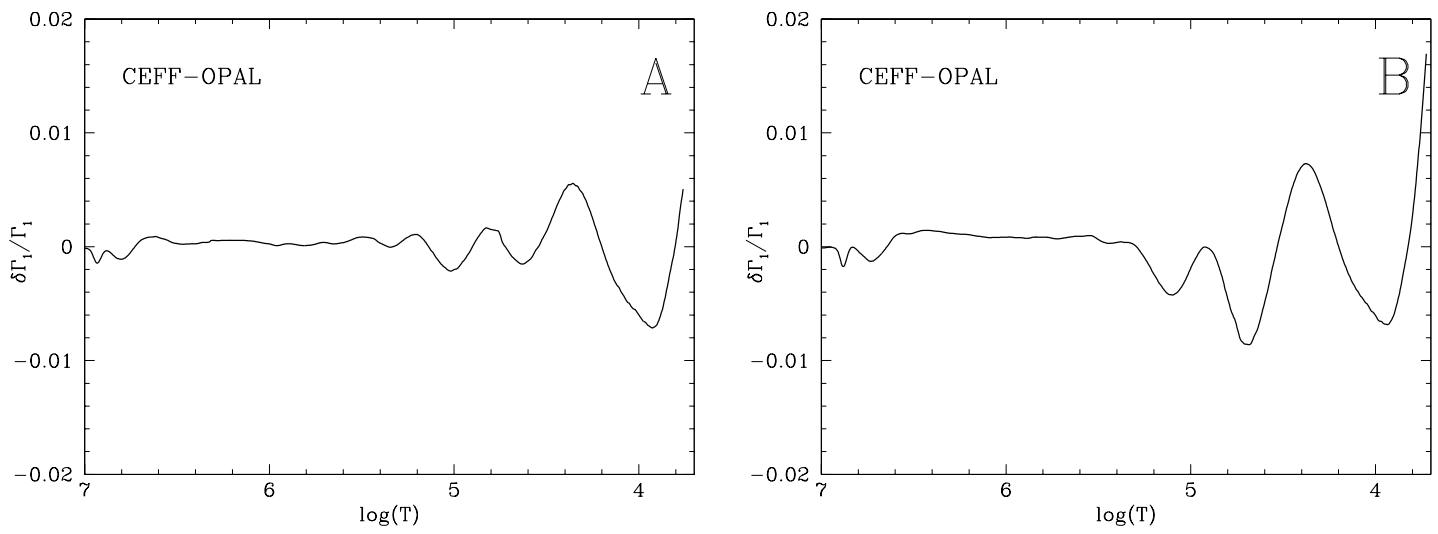

FIGURE 5. Intrinsic difference in $\Gamma_{1}$ in model A0 (left panel) and B0 (right panel).
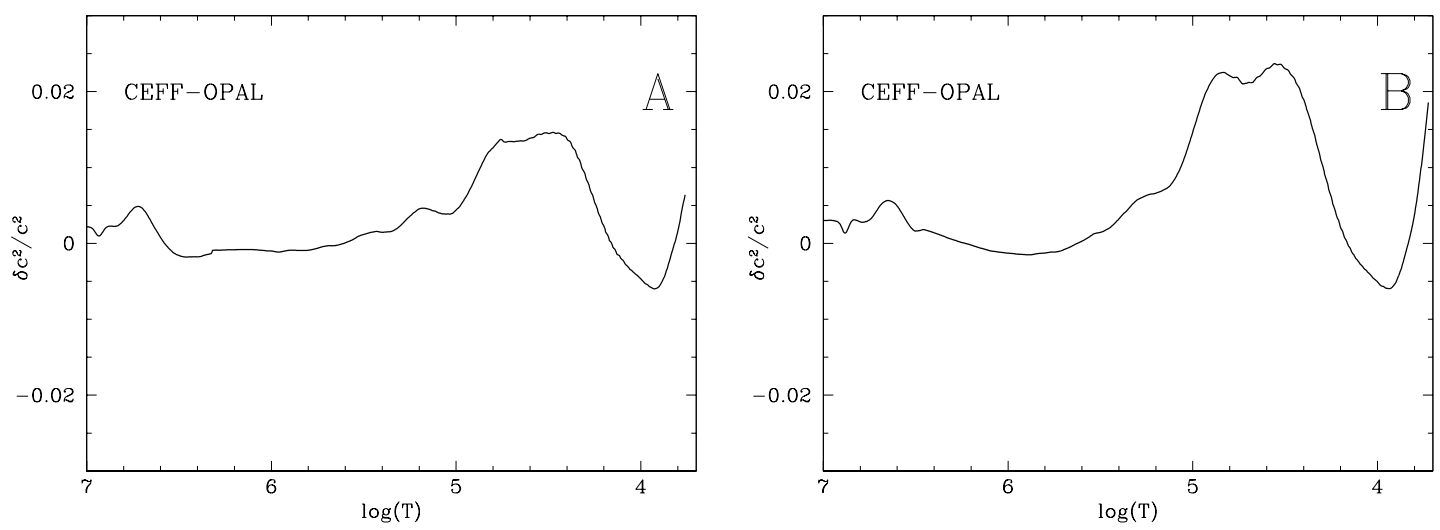

FIGURE 6. Intrinsic difference in squared adiabatic sound speed $c^{2}$. 

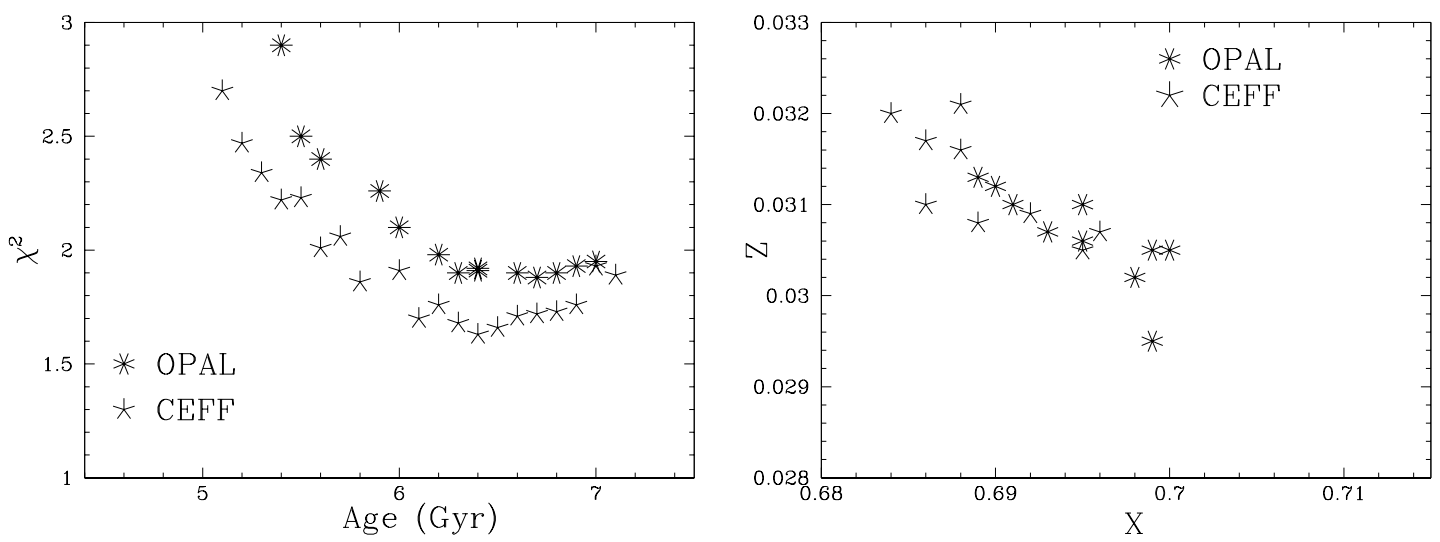

FIGURE 7. $\chi^{2}$ of the best model as a function of age. Initial chemical composition of models calibrated with CEFF and OPAL that have a similar $\chi^{2}$.

\section{CALIBRATION USING OPAL AND CEFF}

Both CEFF and OPAL lead to a quantitatively $\left(\chi^{2}\right)$ similar calibration, nonetheless the difference between model parameters (in particular X,Z and Age) corresponding to good-fit models computed with CEFF and OPAL could represent a useful estimate of systematic uncertainties in the parameters due to the use of a different EOS in the modelling. For this purpose we have run our calibration algorithm with both EOS; the results are shown in Fig. (7) The initial helium content and metallicity of models that have a similar fit to the observational constraints slightly differ (right panel) and no significant difference in the age estimated using CEFF and OPAL calibrations is found (left panel).

\section{CONCLUSIONS}

In agreement with previous works (e.g. [5]) we find that present day accuracy of observed p-mode oscillations in solar-type stars is not sufficient to isolate and detect any "equation of state effects" in the calibration of the binary system $\alpha$ Centauri. We show nonetheless that the comparison between calibrations performed using different equations of state could provide an additional source of systematic uncertainty on the model parameters. 


\section{ACKNOWLEDGMENTS}

The author acknowledges financial support from the Prodex-ESA Contract $15448 / 01 / \mathrm{NL} / \mathrm{Sfe}(\mathrm{IC})$ and is thankful to J. Montalbán, A. Noels and R. Scuflaire for very useful discussions and help in the redaction of the paper. Thanks are also due to M.P. Di Mauro and to the organizers of the meeting.

\section{REFERENCES}

1. Alexander, D. R., Ferguson, J. W. 1994 ApJ 437, 879

2. Basu S. and Christensen-Dalsgaard, J. 1997 A\&A 322, L5

3. Böhm-Vitense, E. 1958, Z. Astrophys., 46, 108

4. Bouchy, F., Carrier, F. 2002 A\&A 390, 205

5. Brown, T. M., Christensen-Dalsgaard, J., Weibel-Mihalas et al. 1994 ApJ 427, 1013

6. Carrier, F. and Bourban, G. 2003 A\&A 406L, 23

7. Caughlan, G. R., Fowler, W. A. 1988, Atomic data and nuclear data tables, 40, 283

8. Christensen-Dalsgaard, J. 1988, in Advances in Helio- and Asteroseismology, eds. J. ChristensenDalsgaard, \& S. Frandsen (Dordrecht: Reidel), IAU Symp., 123, 295

9. Christensen-Dalsgaard, J. and Däppen W. 1992 Astronomy and Astrophysics Review 4, 267

10. Cox, J. P., \& Giuli, R. T. 1968, in Principles of Stellar Structure, eds. Gordon and Breach, Chap. 14

11. Eggenberger, P., Charbonnel, C., Talon, S. et al. 2004 A\&A 417, 235

12. Grevesse N., Noels, A. 1993 La formation des éléments chimiques, eds. B. Hauck, S. Paltani, D. Raboud, AVCP, 205

13. Iglesias, C.A., Rogers, F.J. 1996 ApJ 464, 943

14. Kervella, P., Thévenin, F., Ségransan et al. 2003 A\&A 404, 1087

15. Kurucz, R. L., 1998, http: / / kurucz . harvard.edu/grids.html

16. Miglio et al., in preparation

17. Neuforge-Verheecke, C. and Magain, P. 1997 A\&A 328, 261

18. Pourbaix, D., Nidever, D., McCarthy, C. et al. 2002 A\&A 386, 280

19. Rogers, F. J., Nayfonov, A. 2002 ApJ 576, 1064

20. Salpeter, E. E. 1954 Austral. J. Phys. , 7 , 373

21. Thoul, A., Bahcall, J. N., Loeb, A. 1994 ApJ 421, 828

22. Thoul, A., Scuflaire, R., Noels, A. et al. 2003 A\&A 402, 293 